\title{
La construcción del conocimiento escolar matemático en alumnos de seis escuelas estatales de Lima (una mirada desde los niños)
}

\author{
Construction of mathematical knowledge school students in six schools \\ state of Lima \\ (a look from the kids)
}

\author{
Jaime Aliaga T. ${ }^{1}$, Israel Rivera P., Manuel Cahuana S., Marcos Paico R. \\ Universidad Nacional Mayor de San Marcos, Lima, Perú \\ (RECIBIDO 15-09-2014, ACEPTADO 02-12-2014)
}

\begin{abstract}
RESUMEN
El pobre rendimiento en matemática de los escolares peruanos tendría su origen en los grados elementales y en la construcción subjetiva del conocimiento matemático por parte del niño. La muestra es de 60 niños de tercer grado de primaria de seis instituciones educativas estatales, con alto y bajo rendimiento en matemática. El abordaje metodológico corresponde a la teoría fundamentada. Los datos se recolectaron mediante focus group. Entre los puntos principales sobre el significado del conocimiento matemático están: Las matemáticas se aprenden en la educación formal con los docentes; es preferible tener una profesora a un profesor; en el hogar se encuentra el apoyo para realizar las tareas escolares; en el imaginario familiar las matemáticas y el lenguaje son lo más importante y se vinculan con la capacidad intelectual.
\end{abstract}

Palabras clave: Conocimiento matemático, educación primaria, teoría fundamentada.

\begin{abstract}
The poor performances in Peruvian scholars' mathematics have their origin in the elementary grades and the subjective construction of mathematical knowledge by the child. The sample of 60 children from third grade six state educational institutions with high and low performance in mathematics. The methodological approach corresponds to grounded theory. Data were collected through focus group. Among the main points about the meaning of mathematical knowledge are: the mathematics memorize in the formal education with the educational ones; it is preferable to have a teacher to a professor; in the home he/she is the support to carry out the school tasks; in the imaginary one family the mathematics and the language are the most important thing and they are linked with the intellectual capacity.
\end{abstract}

Keywords: Mathematical knowledge, primary education, grounded theory. 


\section{INTRODUCCIÓN}

En el país una cantidad muy significativa de escolares se encuentran en un nivel muy bajo en el rendimiento en matemática como lo indica, por ejemplo, la Evaluación Censal de Estudiantes 2010 en la que sobre 3 niveles posibles de resultados $(2,1,<1)$, el $57,6 \%$ y el $39,1 \%$ de los alumnos del $2^{\circ}$ de primaria de escuelas estatales y privadas, respectivamente, se ubican en el Nivel $<1$ teniendo dificultades para responder las preguntas más fáciles (UMC, 2010). Creemos que esta situación hace necesario estudios que desde diversas perspectivas epistémicas y metodológicas generen información que contribuya a generar nuevas condiciones para el mejor aprendizaje de la matemática escolar.

La práctica educacional de la matemática escolar adquiere una realidad concreta en el proceso de enseñanza-aprendizaje al que concurren como elementos principales el profesor, el alumno y un contenido, sin olvidar la importancia de otros factores que contextualizan socialmente, psicológicamente, culturalmente e históricamente este proceso, por ejemplo la familia inserta siempre en un determinado estrato socioeconómico. Son variados los estudios que se han ocupado del rol del docente en la construcción del conocimiento escolar (véase p.e. Rodrigo y Arnay, 1997; Melchor y Melchor, 2002), pero son pocos los que han analizado - sobre todo desde una visión cualitativa - al estudiante en el proceso de esta construcción, elemento muy importante pues es el estudiante o sujeto que aprende el que en definitiva aprende bien o mal las matemáticas.

El estudiante es un ser histórico que adviene al nivel educativo primario con una experiencia de vida cotidiana y un conocimiento cotidiano "previo" con su propia epistemología que se conjunciona con la vida cotidiana escolar y un conocimiento escolar que también tiene su propia epistemología (Rodrigo, 1994). Vida y conocimientos escolares cargados de rutinas y símbolos construidos mediante interacciones grupales (Vigotski, 1977) que aluden a procesos de intersubjetividad que en interacción conforman la subjetividad del alumno. Aquí la clave está en la actividad del sujeto por lo que no hay objeto de enseñanza sino de aprendizaje y mediante éste la construcción del conocimiento matemático. Esta actividad consiste principalmente en la elaboración de significados asociados a la propia experiencia, incluida la experiencia lingüística del estudiante con la matemática y con el docente de matemática. Significados que expresados principalmente en creencias, sentimientos, actitudes, valores e ideologías, sirven de base para el desarrollo de acciones voluntarias que intencionalmente desarrolla el individuo para la construcción -buena o no - de los saberes matemáticos escolares.

La cualidad fundamental del ser humano es la de ser sujeto, que comprende un entrelazamiento de múltiples componentes tales como historia personal, características cognitivas, impulsores internos no conscientes, características del sistema nervioso central, estado funcional actual, situación presente, que definen al hombre concreto, real, actuante, al individuo humano de "carne y hueso" (Arias, 1998). A medida que el ser humano adquiere el lenguaje este juega un rol decisivo para que desde niño como individuo-sujeto pueda tomar conciencia de ser consciente 
y la conciencia de sí en forma claramente inseparable de la autoreferencia y la autoreflexividad; lo que le hace posible el objetivarse para luego resubjetivarse en un bucle recursivo incesante (Morín, 2005). En esta recursividad en nuestro parecer se origina la subjetividad, que es la parte más simbólica, más compleja de la psique en el sujeto (Díaz y Gonzales-Rey, 2005), que se constituye en un proceso no lineal en diferentes y simultáneos niveles.

La subjetividad se construye como forma de lo real y constituye un sistema que se externaliza permanentemente objetivando lo subjetivo, y actúa sobre la propia subjetividad que lo produjo desde una nueva dimensión, así la subjetividad se constituye en el curso de su propia historia, social, cultural, por su naturaleza, pero irreductible a su origen, una vez constituida en su propia dimensión histórica. Lo social, lo cultural, se constituye en subjetivo en la propia historia del sujeto individual y en sus diferentes formas de relación, pero lo externo sólo actúa sobre el sistema en la medida en que se subjetiviza, y entra en los propios términos constitutivos del sistema (Gonzales Rey, 1999); simultáneamente se expresa a nivel social como constituyente de la vida social en la forma de subjetividad social que no se diferencia de lo individual por su origen, sino por el escenario de su constitución (Díaz y Gonzales Rey, 2005).

Gonzales-Rey define a la subjetividad como la "organización de los procesos de sentido y significado que aparecen y se configuran de diferentes formas en el sujeto y en la personalidad, así, como en los diferentes espacios sociales en los que el sujeto actúa" (1999:108). La subjetividad constituye un sistema en el que la personalidad tiene el momento organizativo de la subjetividad individual, pero ésta no se reduce a la personalidad porque es una subjetividad que existe en un sujeto en acción, en un sujeto en relación y por lo tanto permanentemente confrontada en un contexto. En ese sentido, la configuración subjetiva es una organización de sentidos subjetivos que definen los procesos simbólicos y las emociones integrados inseparablemente en relación a las experiencias del sujeto dentro de los espacios simbólicos de la cultura, por ejemplo el aula de clase. Corresponde a la personalidad definir una presencia de sentido subjetivo en el marco actual de actuación del sujeto, presencia que no es causa del comportamiento sino un momento de producción de sentido subjetivo que anticipa la acción del sujeto y que se integra a ella dentro de un proceso con múltiples alternativas de desarrollo que puede tener formas muy diferentes de expresión (Díaz y Gonzales Rey, 2005).

En el momento de la indagación de los sentidos subjetivos se elaboran por el sujeto representaciones a través del lenguaje que están influidas por los discursos sociales, los valores y las representaciones. En otras palabras, los procesos de sentido y significado aluden a procesos conocidos como representaciones mentales, identidad, autoestima, valores, motivación (Mitjans, 2008), y otras categorías como las de conocimiento cotidiano previo y epistemología cotidiana previa que mediante los intercambios sociales elabora el individuo en la experiencia vivenciada de la vida diaria (Villegas y Gonzales, 2011), las que al acceder el niño a la escuela interaccionan con ideas construidas que dan forma a la vida cotidiana escolar, al conocimiento cotidiano escolar con su epistemología (Rodrigo, 1994). 
En este contexto, es foco de interés en esta investigación la construcción del conocimiento matemático averiguando los sentidos y significados que los niños otorgan al aprendizaje de la matemática al elaborar desde su perspectiva un marco sobre el proceso de construcción del conocimiento matemático escolar. Una manera de abordar el estudio de los sentidos y significados es empleando una metodología cualitativa, en la que epistémicamente el conocimiento de la realidad es un producto de la actividad humana, por lo tanto se produce en lugar de descubrirse (Pérez, 2003). En consecuencia, el objeto de la metodología cualitativa es el conocimiento de la realidad y su finalidad es comprender las complejas interacciones que se dan en las realidades, por lo que es de su interés identificar, captar, contextualizar, los sentidos y significados, es decir, según Báez (2009), las imágenes sociales, las representaciones sociales, las actitudes, las motivaciones de los sujetos.

La metodología cualitativa es conveniente para conocer las razones por las que un individuo o grupo, piensa, siente o actúa de la forma en que lo hace, sea en lo cotidiano o en el caso de un suceso que puede dar lugar a cambios en la percepción que se tiene de las cosas. Entre sus diversas características resalta su interés en comprender la conducta humana desde el propio marco de referencia del que actúa; el aceptar que existen varias interpretaciones de la realidad, pero también que algunas tienen mayor poder explicativo y son más verosímiles que otras interpretaciones; el adherirse a conocer las razones por las que sucede lo que sucede (Cock y Reichardt, 1986; Báez, 2009; Bautista, 2011); y el que sus resultados permanezcan relativamente estables en el tiempo ya que las motivaciones profundas, los juicios, las actitudes, las representaciones tienden a ser estables, consistentes, pudiendo surgir el cambio por la acción de largo procesos personales y de la evolución propia de las sociedades o por un cambio brusco debido a un hecho puntual (Báez, 2009).

La sustancia y herramienta de la metodología cualitativa es el lenguaje, pues en el procedimiento fundamental se interroga a las personas para que narren las realidades tal como ellas las perciben, elaborando para el efecto su propio discurso empleando recursos narrativos - textos producidos en una situación de comunicación interpersonal, sea oral, escrita, filmada, dibujada - que exponen, revelan, aclaran, glosan, puntualizan, relatan, ejemplifican, las maneras en que se da esa comprensión (Báez, 2009). En el proceso de aprehender el sentido de lo subjetivo el investigador analiza lo que se dice y cómo se dice (contenido) y los recursos usados para comunicarlo.

Lo dicho en el párrafo anterior corresponde a la metodología cualitativa en general, pues la misma no constituye un enfoque monolítico pues en su interior existen varias perspectivas de investigación cada una con su orientación metodológica, supuestos teóricos y conceptuales de la realidad. Una de las perspectivas más importantes es la Teoría Fundamentada (Grounded Theory) (Flick, 2002, en Vasilachis, 2006), que en términos generales, permite en forma inductiva en el proceso de investigación generar conceptos e interrelacionarlos, siguiendo un conjunto de reglas generadas por sus autores los sociólogos estadounidenses 
Barney Glasser y Anselm Strauss en 1967, quienes posteriormente desarrollaron sus propias estrategias metodológicas.

Brevemente descrita esta metodología utiliza, entre otras técnicas de colecta de datos, a la entrevista y el grupo focal (focus group). La información generada se analiza a través del método de la comparación constante, que consiste en comparar la información obtenida tratando de buscar lo común en el conjunto de datos que comparten una misma idea. Esta operación recibe el nombre de codificación y supone el leer y releer los datos para descubrir relaciones, vale decir, el codificar es el inicio de la interpretación (Soneira, 2006, en Vasilachis, 2006). La codificación es de varios tipos: 1) Codificación de las unidades de análisis (codificación abierta), se revisa la información proporcionada, por ejemplo, por las entrevistas y el focus group, generándose en función de sus características y propiedades conceptos y categorías respecto del fenómeno estudiado. El análisis se mantiene como provisional y se plantean preguntas y proposiciones (hipótesis) sobre el fenómeno (Strauss y Corbin, 2002); 2) Se describen las categorías encontradas en primer nivel, se reorganizan los datos fragmentados en la fase anterior estableciendo conexiones entre categorías y subcategorías agrupándolos en temas y patrones (codificación axial); y, 3) Se genera teorías, explicaciones (codificación selectiva). Es un proceso complejo en donde se integran y refinan las categorías y sus relaciones bajo un eje o categoría central, este proceso permite integrar conceptos y categorías de forma sistemática para llegar finalmente a la comprensión del fenómeno estudiado.

La formación de categorías se basa en el criterio de "semejanza familiar" para la formación de conceptos propuesto por Rosch (Hernández, Fernández y Baptista, 2010). A la "semejanza familiar" se llega mediante un proceso de comparación en el que se maximizan las similitudes y se minimizan las diferencias para luego enfatizar las diferencias y minimizar similitudes. En este proceso se hace énfasis en que las categorías deben surgir de los datos y ser fácilmente aplicables a ellos, asimismo deben ser significativamente apropiadas y capaces de explicar el aspecto en estudio. El proceso de comparación permite delimitar la teoría que se caracteriza por hacer máxima la explicación y comprensión de lo estudiado con el mínimo de conceptos y formulaciones, lo que implica la reducción de categorías, y por buscar ampliar el campo de aplicación de la teoría sin desligarse de su base empírica. La reducción de categorías conduce a la elaboración de una categoría central que aparece con alta frecuencias de menciones. Al identificarse una categoría central se interrumpe el procedimiento de codificación de información no conectada con la categoría. Se codifica solo para la categoría central (codificación selectiva). Llega un punto de la recolección de la información que no se agrega nuevo conocimiento sobre esta categoría y sus propiedades o atributos, en consecuencia se interrumpe la codificación para esta categoría ya que se ha producido una saturación de la categoría.

Es pertinente señalar que la teoría generada es de rango medio no debiéndosele confundir con las teorías formales científicas. La teoría de rango medio, también 
denominada sustantiva por sus autores, está referida una misma clase de grupos o casos, que en nuestro caso son los sentidos subjetivos expresados en representaciones de los niños respecto de la construcción de su conocimiento matemático. Las representaciones aluden a sistemas de nociones, prácticas, valores, que permiten orientarse a los niños en ese contexto.

\section{MÉTODO}

Tipo y diseño de investigación: La investigación se inscribe en el paradigma cualitativo con un diseño sustentado en algunos de los principios de la Teoría Fundamentada (Grounded Theory) o Metodología de la Comparación Constante (MCC), denominada sustantiva o de rango medio para diferenciarla de las Teorías Formales, cuya perspectiva es mayor (Glasser y Strauss, 1996; Hernández, Fernández y Baptista, 2010). Se aplicó para generar categorías conceptuales, propiedades y criterios con base en los sentidos subjetivos que los niños conceden al aprendizaje de las matemáticas y a la relación con el profesor. Básicamente se empleó los procesos de codificación abierta y axial y el de saturación teórica.

Participantes: En la selección de los participantes, se utilizó un muestreo no probabilístico de sujetos-tipo, para garantizar la riqueza, profundidad y calidad de la información (Hernández, Fernández y Baptista, 2010). El criterio de inclusión fue el de tener un buen rendimiento o un bajo rendimiento en matemática, condición que fue informada por el profesor de aula. Se seleccionaron 60 estudiantes del tercero de primaria de seis instituciones educativas estatales de educación primaria ubicadas en los distritos de San Juan de Lurigancho, Comas y Ventanilla.

Técnicas: En la recolección de los datos se empleó el grupo focal o focus group. Se realizaron seis focus group. En los primeros cuatro participaron 12 alumnos en cada uno, la mitad con alto rendimiento en matemáticas y la otra mitad con bajo rendimiento además hubo igual número de varones y de mujeres. En base a lo observado respecto de la productividad de estos grupos, se decidió que el quinto grupo estuviera conformado por alumnos con promedio alto, contándose con 6 participantes (4 mujeres y 2 hombres); el sexto grupo fue formado por niños con promedio bajo, participando 3 mujeres y 3 hombres. Los focus group se realizaron en una sola sesión con una duración máxima de 50 minutos. Cada sesión fue organizada de manera que hubiera un líder y un asistente. El primero orientaba la dinámica y utilizaba las frases inductoras y, algunas veces, dibujos, en relación al aprendizaje y el profesor de matemática. Las sesiones fueron filmadas y grabadas. Las grabaciones fueron transcritas para su análisis mediante la teoría fundamentada.

\section{RESULTADOS}

El análisis de los sentidos subjetivos de los niños expresados en las representaciones que tienen de la construcción del conocimiento matemático escolar indica que esta construcción, por lo menos en el nivel del tercer grado de primaria, se daría en relación a diversos aspectos y factores. Veamos: 


\section{El ámbito educativo y el rol docente}

Lo manifestado por los niños y niñas hacen referencia a que su proceso de aprendizaje de la matemática se da esencialmente en el colegio, es decir en el ámbito de la educación formal; asimismo, que en este proceso se da una dinámica unidireccional profesor a alumno, donde el aprender implica adquirir conocimientos o destreza en el manejo de las operaciones matemáticas, yen el que el docente juega un rol principal mientras que los niños y niñas asumen un rol pasivo, el de simples oyentes. El docente se constituye en un factor que juega un rol fundamental en su aprendizaje. Al docente le son atribuidas varias características necesarias de tener por él: método, trato, paciencia, generador de un clima de aprendizaje. Los niños indican que los docentes que no se muestran pacientes, que los golpean y que les gritan, los llevan a un estado emocional desagradable verbalizado a través de "nos sentimos mal"; sentir característico de un sentimiento ansioso que según la abundante literatura científica al respecto, afecta la capacidad de atención-concentración de los alumnos y también su estado cognitivo lo que redunda negativamente en su rendimiento (p.e. Aliaga, Ponce, Bernaola y Pecho, 2000). Estado emocional que interfiere con su aprendizaje, pero que, a la vez, los lleva a estar pendientes de su actuar para evitar las conductas negativas, de castigo, por parte del maestro. Por otro lado, un clima de aprendizaje negativo originado en el grito, el castigo, lleva a la desconfianza en el niño, a que este tenga muchas dudas respecto del tema tratado que no expresa por temor, y a que no vea a su profesor como un apoyo a nivel personal. Al respecto, creemos que si los docentes pusieran en juego un eros pedagógico (Cusianovich, 2005) la percepción que el niño tiene del accionar del profesor en el aula mejoraría notablemente.

Los niños plantearon el perfil de su maestro ideal, señalando en primer lugar su preferencia por el sexo femenino en oposición al sexo masculino. Los profesores varones tienen una imagen a la cual atribuyen características negativas tales como el tener poca paciencia y ser violentos, las profesoras, en contraparte, "tienen paciencia", hablan "bonito", tienen buen trato, no insultan, tienen buena letra ("bonita y clara") y tienen juguetes o hacen dinámicas lúdicas ("juegos").

El preferir a las profesoras sobre los profesores tiene un significado referido a la simbolización, a cómo construyen los niños en sus imaginarios la cuestión referida al género. No es a la mujer en sí misma a la que se desea como profesora, sino a una mujer con determinados comportamiento de género: cuidado, delicadeza, suavidad, paciencia, sinceridad, confiabilidad, creatividad (Ragúz, 1995). Para los niños una profesora con estas características simboliza la garantía de un clima adecuado para el aprendizaje de la matemática, de una seguridad emocional, de libertad y confianza para opinar y plantear dudas. Desde el punto de vista de la teoría del género, los profesores varones con "masculinidad interna" (Ragúz, 1995), cuyo comportamiento no excluya ciertos valores y prácticas entendidos como femeninos, por ejemplo, "suavidad en el trato", pudieran ser también preferidos como profesores de matemática. 


\section{El ámbito familiar}

El ambiente familiar es visto por los niños como secundario respecto del contexto escolar en la construcción del conocimiento matemático, del aprendizaje matemático, pero no por ello deja de tener un rol importante en dicha construcción. Los niños consideran que en sus hogares se tiene la idea que el estudio escolar constituye una actividad "más útil" que los juegos o pasatiempos. Asimismo, que es allí donde reciben el acompañamiento en la resolución de sus tareas escolares y el refuerzo o castigo según el éxito o fracaso en el rendimiento en la materia. También estiman que en el imaginario familiar los cursos de matemática y de lenguaje son los más importantes y que son vinculados a la inteligencia y a capacidad intelectual. La vinculación con la inteligencia o capacidad puede en muchas familias fortalecer la idea que las matemáticas son difíciles y que solo algunos tienen dominio de ellas, lo que constituye ideas negativas porque los niños asimilan la idea que las matemáticas son complicadas y que no hay mucho problema si no las dominan, lo cual pudiera llevarlos a no esforzarse, y en consecuencia a un rendimiento pobre.

\section{Propósito del aprendizaje de las matemáticas}

Otro aspecto relevante identificado en nuestro estudio es el propósito que los niños le otorgan al aprendizaje de la matemática. Esta es vista como básicamente práctica, es decir como una materia que enseña el modo de hacer algo, enseñanza muy relacionada con lo lúdico; un saber útil pero concretizado en "saber contar", "saber hacer operaciones", por sí mismas. En poquísimos casos se percibe la aplicabilidad de las matemáticas expresada, por ejemplo, en "sirve para comprar y para que no te engañen en las compras".

Por otro lado, en relación con el propósito del aprendizaje de la matemática, existen una serie de actividades "universales" que han dado pie a la construcción de las matemáticas y están presentes en todas las culturas, éstas son: contar (el uso de una forma sistemática para comparar y ordenar fenómenos discretos); localizar (exploración espacial del entorno cercano, conceptualización y simbolización de dicho entorno a partir de modelos, diagramas, palabras, etc.); medir (cuantificación de la cualidad para la mayor parte de las comparaciones y ordenaciones, usando objetos o señales como instrumentos de medida); diseñar (creación deformas para los distintos objetos o para cualquier parte de un entorno espacial); jugar (inventar juegos y pasatiempos divertidos, con más o menos reglas); explicar (buscar formas de justificar la existencia de fenómenos que pueden ser religiosos, animistas, científicos, etc.). Las capacidades que deben desarrollar los niños del tercer grado de primaria en el área matemática están contenidas en el Diseño Curricular Nacional (DCN), las que según el grado están relacionadas con todas estas actividades "universales"; pues bien, los niños participantes del estudio se han referido a solo tres aspectos: contar-mayoritariamente-, medir y jugar, quedando postergados el localizar, diseñar y explicar, que son actividades con mayor estructuración o abstracción. Lo que indicaría que no se están desarrollando todas las capacidades matemáticas que deberían desarrollarse en este grado. 


\section{Actitud de los alumnos hacia las matemáticas}

Los niños participantes expresaron actitudes ambivalentes hacia las matemáticas, definiéndolas como "divertidas", pero "complejas" y "aburridas". Una actitud es una predisposición aprendida, no innata, y estable aunque puede cambiar, a reaccionar de una manera valorativa favorable o desfavorable, ante un objeto (Katz 1960 y Zimbardo, 1969, en Morales, 2006); objeto que en nuestro caso es la matemática. Una actitud positiva hacia las matemáticas refleja básicamente una disposición emocional positiva con respecto a la misma, de una manera similar, una actitud negativa se relaciona a una disposición emocional negativa hacia la matemática. En esta ambivalencia probablemente estén involucradas las representaciones negativas que muchos niños tienen acerca del maestro de matemáticas, a quienes, como dijimos anteriormente, si son varones, perciben como aburridos, agresivos física y psicológicamente ("golpean" y no "les tienen paciencia”).

\section{Imaginarios sociales sobre las matemáticas}

Los niños y niñas reproducen en su imaginario a las matemáticas como un medio/ instrumento de éxito, como medio para ser un profesional competente, - que dicho sea de paso es un imaginario social en nuestro país y de la sociedad en general-. Además, también hay una representación social, una lógica de entendimiento desde lo numérico-racional: las matemáticas como sinónimo de inteligencia y superioridad. A este imaginario se asocia la alegría o la tristeza por el conocimiento o desconocimiento de los contenidos matemáticos. Respecto de esta asociación, se evidencia que la "actitud", la "motivación" y en general la "afectividad", ejercen influencia en la construcción del conocimiento matemático de los niños y las niñas (Gómez, 2002). En estudiantes peruano se ha encontrado que la actitud hacia la matemática contribuye notablemente al rendimiento en matemática incluso en el mismo nivel o algo más que la inteligencia general (Aliaga y Frisancho, 2012).

\section{CONCLUSIONES}

1. Para los niños y niñas la educación formal - la escuela - sería el espacio donde se adquiere el conocimiento matemático, mientras que la familia o el hogar es un lugar de apoyo o de complementación de dicha adquisición.

2. En relación al docente, más que el grado de conocimiento de los docentes sobre la materia, es fundamental para la construcción del conocimiento matemático una metodología dinámica, creativa y la manera de relacionarse con sus alumnos. La capacidad para relacionarse positivamente con los estudiantes es esencial para el proceso de construcción del conocimiento matemático escolar.

3. El docente varón es percibido negativamente por la mayoría de los alumnos, prefiriéndose a las docentes por las características que los niños les atribuyen y que corresponden a las características también atribuidas por la sociedad. En esta preferencia están implicado el género. Es posible que un docente varón de "masculinidad interna" pueda tener el mismo grado de preferencia por los niños. 
4. Las representaciones sociales o el imaginario social sobre las matemáticas y en general sobre la educación, es parcializado ya que se privilegia dos cursos: matemática y lenguaje; lo cual va en perjuicio de otras capacidades o inteligencias. En especial, al rendimiento en matemática se le adscribe el ser demostrativo de capacidad intelectual.

5. El conocimiento en matemática es considerado por los niños y niñas como práctico y útil, pero se concreta en el "saber" operaciones básicas, no percibiendo su aplicabilidad en las situaciones de la vida cotidiana.

6. En conexión con lo anterior, los niños no han desarrollado todas o en gran parte las capacidades a adquirir en el tercer grado según el DCN, se han reducido al contar, medir y jugar.

7. La actitud de los niños y niñas hacia las matemáticas no es positiva ni negativa, es ambivalente.

8. El conocimiento matemático es un medio para obtener éxito profesional y es prácticamente equivalente a capacidad y superioridad intelectual.

\section{NOTA DE RECONOCIMIENTO}

Nuestro mayor agradecimiento al psicólogo Rubethson Deyvit Rondan Sáenz y los internos de psicología Jakelin Karina Ruiz Vilca, Ronald Wilfredo Castillo Blanco y Jaikel Homero Rodríguez Bayona por su apoyo en la ejecución de la presente investigación.

\section{REFERENCIAS BIBLIOGRÁFICAS}

Aliaga. J. y Frisancho, A. (2012). Personalidad, inteligencia y actitud hacia las matemáticas en estudiantes de educación secundaria con alto y bajo rendimiento en matemáticas. En Revista Peruana de Psicología y Trabajo Social. 1 (2), 12-24.

Báez, J. (2009). Investigación cualitativa (2 ed.). Madrid: ESIC.

Bautista N. (2011). Proceso de la investigación cualitativa: Epistemología, metodología y aplicaciones. Colombia: Manual Moderno.

Cook, T.D. y Reichardt, CH.,S. (1986). Métodos cualitativos y cuantitativos en investigación evaluativa. Madrid: Morata.

Cussiánovich, A. (2005). Educando desde una pedagogía de la ternura. Ifejant, Lima. Disponible en: http://www.insumisos.com/lecturasinsumisas/Pedagogia\%20de\%20 la\%20ternura.pdf

Díaz, A. y Gonzales, F. (2005). Subjetividad: Una perspectiva histórico cultural. Conversación con el psicólogo cubano Fernando González Rey. En Universitas Psychologica, 4 (3) Bogotá. Recuperado de http://www.scielo.unal.edu.co/scielo. php?script=sci_arttext\&pid=S1657-92672005000300011\&lng=es\&nrm

Glaser, B. G. y Strauss, A. L. (1996). The discovery of grounded theory. Chicago: Aldine Publishing Company. 
Gómez, J. (2002). De la enseñanza al aprendizaje de las matemática. Barcelona: Paidós.

Melchor, J. y Melchor, V. (2002). El conocimientos de las matemáticas. En: Xixim, Revista Electrónica Didáctica de las Matemáticas, Año 3, №1, pp: 16-29. Universidad Autónoma de Querétaro, México.

Mitjáns, A. (2008). Subjetividad, Complejidad y Educación. Revista Electrónica Internacional de la Unión Latinoamericana de Entidades de Psicología. $N^{\circ} 13$. Recuperado de http://www.psicolatina.org/13/subjetividad.html

Pérez, G. (2003). Investigación cualitativa. Métodos y Técnicas. Buenos Aires: Docencia.

Rodrigo, J.M. (1994). Etapas, contextos, dominios y teorías implicitas en el conocimiento social, en M.J. Rodrigo (Comp.). Contexto y desarrollo social. Madrid: Síntesis.

Rodrigo, M. J. y Arnay, J. (1997). La construcción del conocimiento escolar. Barcelona: Paidós.

Strauss, A. y Corbin, J. (2002). Bases de la investigación cualitativa: Técnicas y procedimientos para desarrollar la teoría fundamentada. Colombia: Universidad de Antioquia.

Villegas, M. y Gonzales, F. (2011). La investigación cualitativa de la vida cotidiana. Medio para la construcción de conocimiento sobre lo social a partir de lo individual. Psicoperspectivas, Vol. 10, N², pp: 35-39. Universidad Pedagógica Experimental Libertador, Venezuela. 